\title{
What Can Be Done to Solve the Unmet Clinical Need of Hepatocellular Carcinoma Patients following Lenvatinib Failure?
}

\author{
Atsushi Hiraoka ${ }^{a}$ Takashi Kumada $^{\text {b }}$ Toshifumi Tada $^{c}$ Kazuya Kariyama ${ }^{d}$ Joji Tani ${ }^{\text {e }}$ \\ Shinya Fukunishi ${ }^{f}$ Masanori Atsukawa ${ }^{g}$ Masashi Hirooka ${ }^{\text {h }}$ Kunihiko Tsuji ${ }^{i}$ Toru Ishikawa $^{\mathrm{j}}$

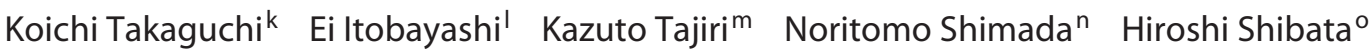 \\ Hironori Ochi $^{p}$ Kazuhito Kawata ${ }^{q}$ Satoshi Yasuda ${ }^{r}$ Hidenori Toyoda $^{r}$ Hideko Ohama $^{f}$ \\ Kazuhiro Nouso $^{d}$ Akemi Tsutsui $^{k}$ Takuya Naganok ${ }^{k}$ Norio Itokawa $^{g}$ Korenobu Hayama ${ }^{9}$ \\ Taeang Arai ${ }^{9}$ Michitaka Imai $^{j}$ Yohei Koizumi ${ }^{h}$ Shinichiro Nakamura ${ }^{c}$ Kouji Joko ${ }^{p}$ \\ Kojiro Michitaka ${ }^{a}$ Yoichi Hiasa ${ }^{\text {h }}$ Masatoshi Kudos RELPEC Study Group and HCC 48 Group \\ ${ }^{a}$ Gastroenterology Center, Ehime Prefectural Central Hospital, Ehime, Japan; 'bepartment of Nursing, Gifu Kyoritsu \\ University, Ogaki, Japan; 'Department of Internal Medicine, Himeji Red Cross Hospital, Hyogo, Japan; ${ }^{\mathrm{d} D e p a r t m e n t}$ \\ of Gastroenterology, Okayama City Hospital, Okayama, Japan; 'Department of Gastroenterology and Hepatology, \\ Kagawa university, Kagawa, Japan; fDepartment of Gastroenterology, Osaka Medical College, Osaka, Japan; \\ 'Division of Gastroenterology and Hepatology, Department of Internal Medicine, Nippon Medical School, Tokyo, \\ Japan; 'Department of Gastroenterology and Metabology, Ehime University Graduate School of Medicine, Ehime, \\ Japan; 'Center of Gastroenterology, Teine Keijinkai Hospital, Sapporo, Japan; 'Department of Gastroenterology,

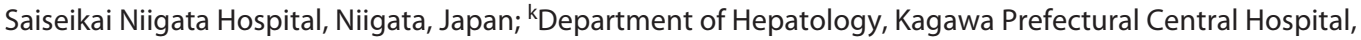 \\ Takamatsu, Japan; 'Department of Gastroenterology, Asahi General Hospital, Asahi, Japan; mepartment of \\ Gastroenterology, Toyama University Hospital, Toyama, Japan; n'Division of Gastroenterology and Hepatology, \\ Otakanomori Hospital, Kashiwa, Japan; 'Department of Gastroenterology, Tokushima Prefectural Central Hospital,

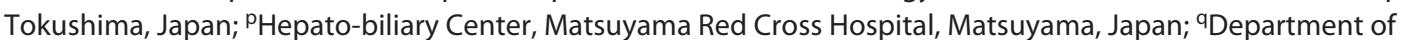 \\ Hepatology, Hamamatsu University School of Medicine, Hamamatsu, Japan; 'Department of Gastroenterology and \\ Hepatology, Ogaki Municipal Hospital, Gifu, Japan; 'Department of Gastroenterology, Kindai University, Osaka, Japan
}

\section{Keywords \\ Hepatocellular carcinoma $\cdot$ Lenvatinib $\cdot$ Hand-foot skin reaction $\cdot$ Postprogression treatment $\cdot$ Beyond progression of disease}

\begin{abstract}
Background/Aim: An effective postprogression treatment of lenvatinib (LEN) against unresectable hepatocellular carcinoma (u-HCC) has not been established. We aimed to elucidate the clinical role of continuing LEN beyond progression of disease (PD). Methods: From March 2018 to October 2020, 99
\end{abstract}

u-HCC patients, in whom PD was confirmed (male:female = 78:21, median age 72 years, Child-Pugh $A=99$, Barcelona Clinic Liver Cancer stage A:B:C $=2: 43: 54$, LEN as first-line $=55$ ), were enrolled (stopped LEN at PD [A group], $n=26$; continued LEN beyond PD [B group], $n=73$ ). Radiological response was evaluated with RECIST 1.1. Clinical features and prognostic factors for overall survival (OS) were retrospectively investigated using inverse probability weighting (IPW) calculated by propensity score. Results: Median time to progression, best response, and modified albumin-bilirubin grade (mALBI) at both baseline and PD did not show significant difference between the groups. Postprogression treatment in the A group
C 2021 The Author(s)

Published by S. Karger AG, Basel

This article is licensed under the Creative Commons AttributionNonCommercial-NoDerivatives 4.0 International License (CC BY NC-ND) (http://www.karger.com/Services/OpenAccessLicense) Usage and distribution for commercial purposes as well as any distribution of modified material requires written permission.
Atsushi Hiraoka

Gastroenterology Center

Ehime Prefectural Central Hospital

Kasuga-Cho 83, Matsuyama, Ehime 790-0024 (Japan)

hirage@m.ehime-u.ac.jp 
was best supportive care in 17 , sorafenib in 4 , regorafenib in 3 , ramucirumab in 1 , and hepatic arterial infusion chemotherapy in 1 . After adjusting with IPW, the B group showed better prognosis in regard to OS after PD and OS after introducing LEN than the A group (10.8/19.6 vs. 5.8/11.2 months, $p<0.001$, respectively). In IPW-adjusted Cox hazard multivariate analysis, significant prognostic factors for OS after PD were mALBI $2 \mathrm{~b} / 3$ at PD (HR 1.983, $p=0.021$ ), decline of Eastern Cooperative Oncology Group performance status (ECOG PS) from baseline at PD (HR 3.180, $p<0.001)$, elevated alpha-fetoprotein $(\geq 100 \mathrm{ng} / \mathrm{mL})$ at introducing LEN (HR 2.511, $p=0.004)$, appearance of new extrahepatic metastasis (HR 2.396, $p=$ 0.006), positive for hand-foot skin reaction (HFSR) before PD (any grade) (HR 0.292, $p<0.001$ ), and continuing LEN beyond PD (HR 0.297, $p<0.001)$. Conclusion: When ECOG PS and hepatic reserve function permit, continuing LEN treatment beyond PD, especially in u-HCC patients showed HFSR during LEN treatment, might be a good therapeutic option, at least until a more effective drug as a postprogression treatment after LEN failure is developed.

\footnotetext{
(c) 2021 The Author(s)
}

Published by S. Karger AG, Basel

\section{Introduction}

Hepatocellular carcinoma (HCC) is the most common primary malignancy of the liver and fifth most common malignancy worldwide [1]. Sorafenib (SOR) [2] and regorafenib (REG) [3] have been developed as powerful molecular targeting agents (MTAs) for patients with unresectable HCC (u-HCC), and Terashima reported that postprogression survival was highly correlated with overall survival (OS) in advanced HCC patients treated with SOR [4]. Prior to introduction of lenvatinib (LEN) in March 2018 [5], there had been no established postprogression treatment protocol for use after REG failure and no treatment established for patients who showed SOR intolerability. Since that time, LEN has been shown in clinical practice in Japan to be useful not only as first-line but also as second- and even third-line treatment $[6,7]$. Nevertheless, establishment of new effective postprogression treatments are needed to obtain better improvement of prognosis for patients with u-HCC. At the time of writing, though ramucirumab (RAM) $[8,9]$ become approved in Japan as an additional MTA drug in June 2019, no other effective postprogression treatment after LEN failure has been established. The present study aimed to elucidate the clinical role of continuing LEN beyond first progression of disease (PD) as an alternative therapeutic method in real-world clinical practice.

\section{Materials and Methods}

This is a report of multicenter analysis results of $183 \mathrm{u}-\mathrm{HCC}$ patients with serial radiological assessment imaging data obtained from March 2018 to October 2020 at 11 different institutions (Ehime Prefectural Central Hospital, Himeji Red Cross Hospital, Okayama City Hospital, Kagawa Prefectural Central Hospital, Kagawa University Hospital, Saiseikai Niigata Hospital, Matsuyama Red Cross Hospital, Nippon Medical School Hospital, Osaka Medical College, Hamamatsu University School of Medicine Hospital, and Toyama University Hospital) (Fig. 1). After confirming no significant differences between time to progression (TTP) determined with Response Evaluation Criteria in Solid Tumors (RECIST), version 1.1 [10], and modified RECIST (mRECIST) [11] in the entire cohort (see online suppl. Fig. 1; for all online suppl.material, seewww.karger.com/doi/10.1159/000513355), RECIST was used for all of the analyses. Of the 183 patients, the clinical features of 99, in whom PD was confirmed by both RECIST and mRECIST, were finally analyzed in a retrospective manner after exclusion of those in whom LEN was discontinued 1 month or more before the time of PD determined by RECIST 1.1, or those without assessment of therapeutic response with an enhanced imaging modality. The 99 patients were divided into 2 groups, those for whom LEN was abandoned at PD (A group: $n=26$ ) and those who continued LEN beyond PD (B group: $n=73$ ) (Fig. 1). Treatment "beyond PD" was defined as LEN treatment continued for $>1$ month after PD by RECIST 1.1. The first assessment of the therapeutic effect was performed using dynamic CT/MRI from 1 to 2 months after introduction of LEN, with imaging examinations performed every 2 months following the initial assessment with dynamic imaging (CT or MRI).

Basal Liver Diseases

HCC due to hepatitis $\mathrm{C}$ virus (HCV) was judged when anti$\mathrm{HCV}$ was positive, whereas HCC due to hepatitis B virus (HBV) was judged when the HBV surface antigen was positive.

\section{Liver Function Assessment}

Child-Pugh classification [12] and albumin-bilirubin (ALBI) grade were used for assessment of hepatic reserve function. ALBI score was calculated with serum albumin and total-bilirubin values using the following formula: ALBI score $=\log _{10}$ bilirubin $(\mu \mathrm{mol} / \mathrm{L}) \times$ $0.66+$ albumin $(\mathrm{g} / \mathrm{L}) \times-0.085(\leq-2.60$, ALBI grade $1 ;>-2.60$ to $\leq-1.39$, grade 2 ; and $>-1.39$, grade 3 ) [13-15]. To perform more detailed evaluations of patients with the middle ALBI grade of 2 , we used a revised grading system consisting of 4 levels that included subgrading for the middle grade of 2 ( $2 \mathrm{a}$ and $2 \mathrm{~b}$ ) based on an ALBI score of -2.27 as the cutoff, which was previously developed based on the value for indocyanine green retention after 15 min of $30 \%[16,17]$.

\section{HCC Diagnosis and Treatment}

Based on an increasing course of alpha-fetoprotein (AFP), as well as dynamic CT [18] or MRI [19, 20], contrast enhanced ultrasonography with perflubutane (Sonazoid ${ }^{\circledR}$; Daiichi Sankyo Co., Ltd., Tokyo, Japan) [21, 22], and/or pathological findings, HCC was diagnosed. We used Barcelona Clinic Liver Cancer (BCLC) [23] and tumor node metastasis (TNM) staging, determined as previously reported in a study for TNM staging of HCC conducted by the Liver Cancer Study Group of Japan (LCSGJ) 6th edition [24] (TNM-LCSGJ), to evaluate tumor progression. 


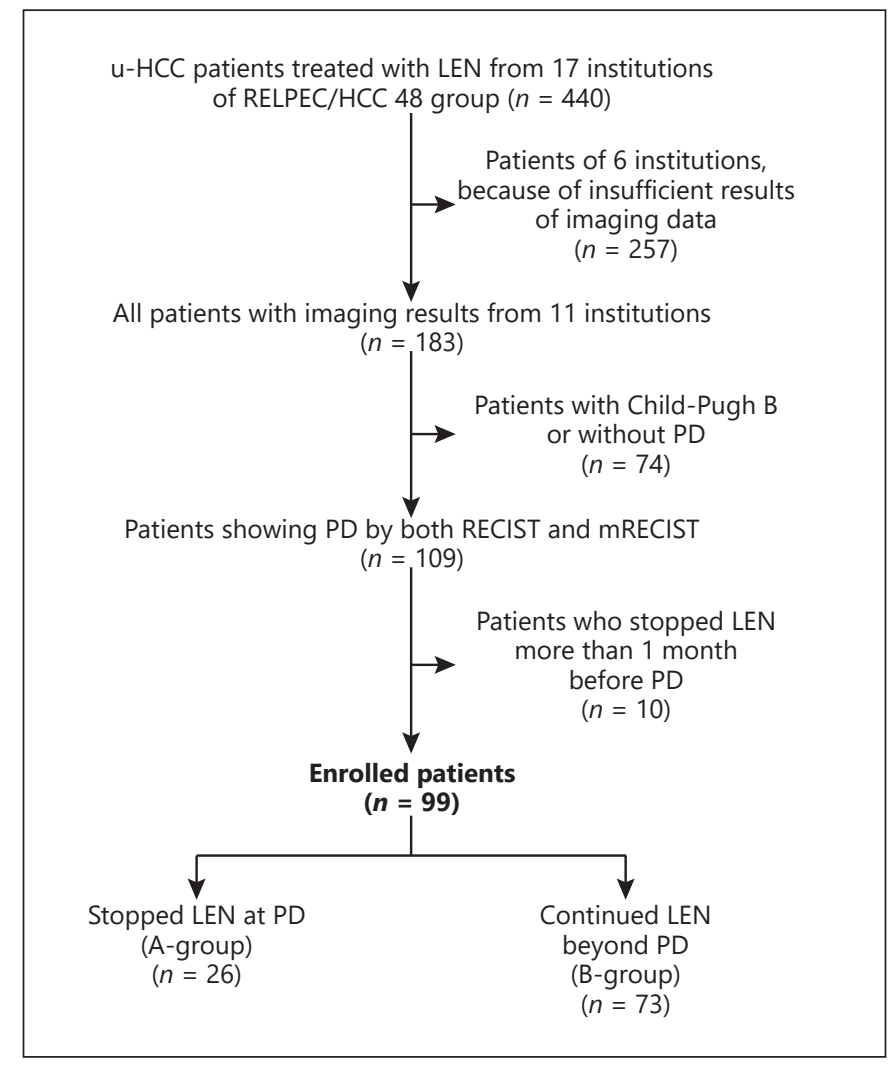

Fig. 1. Flowchart of patient enrollment. u-HCC, unresectable hepatocellular carcinoma; PD, progression of disease; LEN, lenvatinib; RECIST, Response Evaluation Criteria In Solid Tumors version 1.1 .

Lenvatinib Treatment and Assessment of Adverse Events

After obtaining written informed consent from each patient, LEN was started. Oral administrations at $8 \mathrm{mg}$ /day were given to patients weighing $<60 \mathrm{~kg}$ and $12 \mathrm{mg} /$ day to those $\geq 60 \mathrm{~kg}$, as much as possible, and discontinued when any unacceptable or serious adverse event (AE) is reported. When clinical tumor progression was observed, the decision to continue or discontinue LEN treatment was made at the discretion of the attending physicians. According to the guidelines for administration of LEN, the drug dose was reduced or treatment interrupted when a patient developed any grade 3 or more severe $\mathrm{AE}$, or if any unacceptable grade 2 drugrelated AE occurred. AEs were assessed according to the National Cancer Institute Common Terminology Criteria for Adverse Events, version 4.0 [25]. AEs of grade 3 or more were defined as severe, and the worst grade for each AE during the present observation period was recorded. If a drug-related $\mathrm{AE}$ was noted, dose reduction or temporary interruption was maintained until the symptom was resolved to grade 1 or 2 , according to the guidelines provided by the manufacturer.

The last day of LEN treatment was defined as the day of last date of administration of LEN before a clear statement of discontinuation of LEN by attending physicians on the medical record, or the last day of administration of LEN before postprogression treat- ments. In analysis for prognostic factors of postprogression overall survival (OS), we used clinical 17 factors described below for calculating inverse probability weighting (IPW) with propensity score.

This was a retrospective analysis of records stored in a database, and official approval was received based on the Guidelines for Clinical Research issued by the Ministry of Health and Welfare of Japan. All procedures complied with the Declaration of Helsinki. The protocol used in the present study was approved by the Institutional Ethics Committee of Ehime Prefectural Central Hospital (IRB No. 30-66).

\section{Statistical Analysis and Calculations of Propensity Score and} Inverse Probability Weighting

Continuous variables are expressed as median values (firstthird quartile). Statistical analyses were performed using Welch's $t$ test, Student's $t$ test, Fisher's exact test, or Mann-Whitney's U test, as appropriate. Prognosis was analyzed by Cox hazard analysis, the Kaplan-Meyer method, and a log-rank test.

The A- and B-group probabilities (propensity) were calculated using logistic regression analysis with a set of covariates deemed likely to have effects on OS, including patients' condition (elderly [age $\geq 75$ years], gender, infection for chronic hepatitis virus, and history of MTA), hepatic function (Child-Pugh scores at introducing LEN and at time of PD), malignant potential of tumor (elevated AFP data $[\geq 100 \mathrm{ng} / \mathrm{mL}]$ at time of introducing LEN and for 1 month before or after PD), BCLC stages (C or D), which include tumor burden, hepatic function, and Eastern Cooperative Oncology Group performance status (ECOG PS), at introducing LEN and at time of PD, decline of ECOG PS from baseline at time of $\mathrm{PD}$, clinical features during LEN treatment (no response to LEN [best response: $\mathrm{PD}$ ]), 4 types of PD patterns (appearance of new extrahepatic metastasis [EHM], appearance of major portal vein tumor thrombosis [Vp3 and $\mathrm{Vp} 4$ ], intrahepatic new lesion, and increasing size without new lesion), and treatment factors (starting dose of LEN [reduced dose]).

IPW was defined as $1 /$ (propensity score) for the A group and $1 /$ (1-propensity score) for the B group. Hazard ratio (HR) for OS after PD of each clinical factor and OS after introducing LEN and OS after PD were tested using IPW-adjusted Cox hazard analysis or an IPW-adjusted log-rank test, respectively [26, 27].

A $p$ value of $<0.05$ was considered to indicate statistical significance. All statistical analyses were performed using Easy R, version 1.53 (Saitama Medical Center, Jichi Medical University, Saitama, Japan) [28], a graphical user interface for R (The R Foundation for Statistical Computing, Vienna, Austria).

\section{Results}

\section{Time to Progression in $183 \mathrm{u}$-HCC Patients}

In all $183 \mathrm{u}$-HCC patients with imaging results from 11 institutions (male:female $=142: 41$, median age 73 years; Child-Pugh score 5:6:7:8:9:10 = 108:62:11:1:0:1; mALBI 1:2a:2b:3 = 59:52:68:4; BCLC 0:A:B:C:D = 1:6:81:94:1; TNM-LCSGJ I:II:III:IVa:IVb = 2:32:63:16:70) (Fig. 1), the median TTP by mRECIST was 8.4 months, 
Table 1. Clinical features of $\mathrm{u}-\mathrm{HCC}$ patients at first progression of disease $(N=99)$

\begin{tabular}{|c|c|c|c|}
\hline Parameters & $\begin{array}{l}\text { A group } \\
(n=26)\end{array}$ & $\begin{array}{l}\text { B group } \\
(n=73)\end{array}$ & $p$ value \\
\hline \multicolumn{4}{|l|}{ At introducing LEN } \\
\hline Age, years ${ }^{\mathrm{a}}$ & $73(64-78)$ & $72(65-78)$ & 0.924 \\
\hline Gender, male:female & $19: 7$ & $59: 14$ & 0.414 \\
\hline Etiology, HCV:HBV:alcohol:other & $16: 2: 0: 8$ & $30: 14: 12: 17$ & 0.037 \\
\hline BMI, $\mathrm{kg} / \mathrm{m}^{2 \mathrm{a}}$ & $22.4(20.2-25.1)$ & $21.8(19.1-23.9)$ & 0.252 \\
\hline ECOG PS at LEN introduction, 0:1 & $22: 4$ & $69: 4$ & 0.201 \\
\hline Platelets, $\geq 10^{4} / \mu \mathrm{L}^{\mathrm{a}}$ & $13.1(10.2-15.5)$ & $14.2(11.1-17.3)$ & 0.207 \\
\hline AST, U/L & $46(32-63)$ & $39(26-59)$ & 0.118 \\
\hline $\mathrm{ALT}, \mathrm{U} / \mathrm{L}^{\mathrm{a}}$ & $38(22-56)$ & $27(19-44)$ & 0.101 \\
\hline Total bilirubin, $\mathrm{mg} / \mathrm{dL}^{\mathrm{a}}$ & $0.8(0.6-1.0)$ & $0.7(0.5-0.8)$ & 0.023 \\
\hline Albumin, $g / \mathrm{dL}^{\mathrm{a}}$ & $3.8(3.6-4.1)$ & $3.8(3.5-4.1)$ & 0.921 \\
\hline Prothrombin time, $\%^{\mathrm{a}}$ & $86.1(77.0-91.0)$ & $93.0(82.0-102.0)$ & 0.044 \\
\hline $\mathrm{eGFR}, \mathrm{mL} / \mathrm{min} / 1.73 \mathrm{~m}^{2 \mathrm{a}}$ & $71.0(60.5-92.3)$ & $72.4(57.0-81.3)$ & 0.534 \\
\hline $\mathrm{AFP}, \mathrm{ng} / \mathrm{mL}$ at LEN introduction ${ }^{\mathrm{a}}(\mathrm{AFP}<100: \geq 100 \mathrm{ng} / \mathrm{mL})$ & $\begin{array}{l}306.5(11.5-1,898.3) \\
(10: 16)\end{array}$ & $\begin{array}{l}24.0(3.9-473.2) \\
(43: 30)\end{array}$ & $0.034(0.108)$ \\
\hline Started with reduced dose of LEN & $5(19.2 \%)$ & $16(21.9 \%)$ & 1.000 \\
\hline Past history of SOR & $15(57.7 \%)$ & $29(39.7 \%)$ & 0.167 \\
\hline Past history of REG & $5(19.2 \%)$ & $7(9.6 \%)$ & 0.291 \\
\hline ALBI score at LEN introduction ${ }^{\mathrm{a}}$ & $-2.48(-2.30$ to -2.61$)$ & $-2.57(-2.24$ to -2.79$)$ & 0.504 \\
\hline mALBI grade at introducing LEN, $1: 2 \mathrm{a}: 2 \mathrm{~b}: 3$ & $7: 12: 7: 0$ & $34: 19: 20: 0$ & 0.127 \\
\hline Child-Pugh score, 5:6 & $18: 8$ & $52: 21$ & 1.0 \\
\hline Intrahepatic tumor size, none: $<2: 2-5:>5 \mathrm{~cm}$ (maximum) & $2: 2: 16: 6$ & $10: 22: 25: 16$ & 0.043 \\
\hline Intrahepatic tumor, $n$, none:single:multiple & $2: 3: 21$ & $10: 7: 56$ & 0.785 \\
\hline Positive for MVI, \% & $2(7.7)$ & $3(4.1)$ & 0.604 \\
\hline Positive for EHM, \% & $12(46.2)$ & $30(41.1)$ & 0.653 \\
\hline TNM-LCSGJ at LEN introduction, II:III:IVa:IVb & $0: 9: 4: 13$ & $17: 20: 6: 30$ & 0.022 \\
\hline BCLC stage at LEN introduction, A:B:C & $0: 10: 16$ & $2: 33: 38$ & 0.729 \\
\hline Best response (CR:PR:SD:PD) ${ }^{\mathrm{b}}$ & $0: 8: 11: 7$ & $3: 23: 31: 16$ & 0.862 \\
\hline \multicolumn{4}{|l|}{ At time of $P^{b}$} \\
\hline ECOG PS at time of PD, ${ }^{\mathrm{b}} 0: 1: 2: 3$ & $13: 8: 2: 3$ & 53:17:3:0 & 0.015 \\
\hline Frequency of decline of ECOG PS from baseline, \% & $11(42.3)$ & $19(26.0)$ & 0.141 \\
\hline AFP, ng/mL at time of $\mathrm{PD}^{\mathrm{b}}(\mathrm{AFP}<100: \geq 100 \mathrm{ng} / \mathrm{mL})$ & $\begin{array}{l}604.1(16.2-6,227.5) \\
(9: 17)\end{array}$ & $\begin{array}{l}48.5(6.1-522.4) \\
(44: 29)\end{array}$ & $0.016(0.038)$ \\
\hline Delta ALBI score at time of $\mathrm{PD}^{\mathrm{b}}$ from baseline $\mathrm{a}^{\mathrm{a}}$ & $0.24(-0.05-0.69)$ & $0.25(0.01-0.47)$ & 0.691 \\
\hline ALBI score ${ }^{\mathrm{a}}$ at time of $\mathrm{PD}^{\mathrm{b}}$ & $-2.18(-2.19$ to -2.64$)$ & $-2.35(-1.94$ to -2.65$)$ & 0.467 \\
\hline mALBI grade at time of $\mathrm{PD}, \mathrm{b} 1: 2 \mathrm{a}: 2 \mathrm{~b}: 3$ & $8: 4: 11: 3$ & $21: 21: 25: 6$ & 0.571 \\
\hline $\begin{array}{l}\text { Child-Pugh score at time of PD, b 5:6:7:8:9: } \geq 10 \text { (frequency of } \\
\text { Child-Pugh class A, \%) }\end{array}$ & $11: 5: 5: 2: 3: 0(16,61.5 \%)$ & $37: 19: 8: 4: 3: 2(56,76.7 \%)$ & $0.612(0.199)$ \\
\hline TNM-LCSGJ at time of PD, ${ }^{\mathrm{b}}$ II:III:Iva:IVb & $0: 7: 4: 15$ & $8: 23: 6: 36$ & 0.243 \\
\hline BCLC stage at time of PD, A:B:C:D & $0: 3: 20: 3$ & $1: 21: 50: 1$ & 0.034 \\
\hline Postprogression treatment, BSC:SOR:REG:RAM:HAIC & 17:4:3:1:1 & na & na \\
\hline \multicolumn{3}{|l|}{ PD pattern (appearance of new EHM:appearance of MVI: appearance of } & 0.939 \\
\hline Period of LEN administration, months ${ }^{\mathrm{a}}$ & $4.5(2.2-7.5)$ & $11.4(6.8-16.3)$ & $<0.001$ \\
\hline \multicolumn{4}{|l|}{ Difference between time of LEN administration discontinuation and } \\
\hline $\mathrm{PD},{ }^{\mathrm{b}}$ months $\mathrm{a}^{\mathrm{a}}$ & $0(-0.4$ to 0.10$)$ & $4.1(2.3-7.3)$ & $<0.001$ \\
\hline Death during observation period, \% & $19(73.1 \%)$ & $33(45.2 \%)$ & 0.021 \\
\hline Observation period, months ${ }^{\mathrm{a}}$ & $7.5(5.5-12.2)$ & $15.4(10.3-21.0)$ & $<0.001$ \\
\hline $\mathrm{IPW}^{\mathrm{a}}$ & $3.02(2.19-4.29)$ & $1.23(1.10-1.45)$ & $<0.001$ \\
\hline
\end{tabular}

u-HCC, unresectable hepatocellular carcinoma; HCV, hepatitis C virus; HBV, hepatitis B virus; BMI, body mass index; ECOG PS, Eastern Cooperative Oncology Group performance status; AST, aspartate transaminase; ALT, alanine aminotransferase; AFP, alpha-fetoprotein; eGFR, estimated glomerular filtration rate; ALBI, albumin-bilirubin; mALBI, modified ALBI grade; MVI, major portal vein tumor thrombosis (Vp3 and Vp4); EHM, extrahepatic metastasis; BCLC, Barcelona Clinic Liver Cancer stage; EHM, extrahepatic metastasis; TNM-LCSGJ 6th, tumor node metastasis stage by Liver Cancer Study Group of Japan, 6th edition; RECIST, Response Evaluation Criteria In Solid Tumors version 1.1; CR, complete response; PR, partial response; SD, stable disease; PD, progression of disease; BSC, best supportive care; SOR, sorafenib; REG, regorafenib; RAM, ramucirumab; HAIC, hepatic arterial infusion chemotherapy; na, not applicable; LEN, lenvatinib; IPW, inverse probability weighting. ${ }^{a}$ Median values (interquartile range) are shown as numbers, unless otherwise indicated. ${ }^{b}$ Evaluated by RECIST 1.1. 
Table 2. AEs in both groups

\begin{tabular}{|c|c|c|c|c|}
\hline & \multicolumn{3}{|l|}{ Before $\mathrm{PD}^{\mathrm{a}}$} & \multirow{2}{*}{$\begin{array}{l}\text { AEs occurred during } \\
\text { beyond } \mathrm{PD}^{\mathrm{a}} \text { treatmen } \\
\text { with LEN } \\
\text { group B (grade 1:2:3) }\end{array}$} \\
\hline & $\begin{array}{l}\text { group } A(n=26) \\
\text { (grade } 0: 1: 2: 3)\end{array}$ & $\begin{array}{l}\text { group B }(n=73) \\
\text { (grade } 0: 1: 2: 3)\end{array}$ & $p$ value & \\
\hline HFSR & $22: 2: 1: 1$ & $44: 13: 16: 0$ & 0.019 & $1: 2: 0$ \\
\hline Appetite loss & $16: 2: 7: 1$ & $46: 14: 10: 3$ & 0.286 & $6: 7: 0$ \\
\hline General fatigue & $15: 6: 4: 1$ & 52:10:11:0 & 0.227 & $3: 1: 0$ \\
\hline Hypertension & $22: 1: 2: 1$ & $54: 5: 12: 2$ & 0.679 & $1: 0: 0$ \\
\hline Urine protein & $20: 3: 1: 2$ & $55: 6: 7: 5$ & 0.838 & $0: 4: 2$ \\
\hline Abnormality of thyroid function & $17: 4: 4: 1$ & 49:9:14:1 & 0.763 & $1: 1: 1$ \\
\hline Diarrhea & $22: 3: 2: 1$ & $55: 11: 6: 1$ & 0.639 & $2: 3: 0$ \\
\hline Elevation of the levels of $\mathrm{NH} 3$ & $23: 0: 0: 3$ & $67: 1: 1: 4$ & 0.665 & $1: 0: 1$ \\
\hline Hoarseness & 23:3:0:0 & $58: 13: 2: 0$ & 0.754 & 3:0:0 \\
\hline Decreasing the levels of platelet count & 25:0:0:1 & $70: 0: 1: 2$ & 1.000 & None \\
\hline Elevation of the levels of transaminase & $25: 1: 0: 0$ & $71: 1: 0: 1$ & 0.603 & $0: 3: 0$ \\
\hline Other AEs of grade 3 & None & $\begin{array}{l}\text { Body weight loss }(n=1) \\
\text { Fever up }(n=1) \\
\text { Neutropenia }(n=1)\end{array}$ & 1.00 & Fever up $(n=3)$ \\
\hline
\end{tabular}

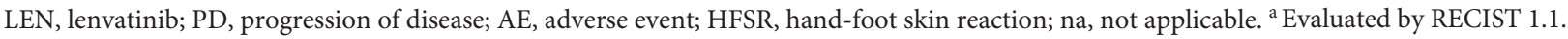

while that by RECIST was 8.1 months (online suppl. Fig. $1 \mathrm{a}, \mathrm{b})$. These results were the same as previously reported by Kaneko et al. [29], in which PD judgment by RECIST and mRECIST showed similar clinical weight in regard to prognosis. Based on the above, we used RECIST 1.1 to evaluate using common criteria, as previously reported in trials of SOR [2], REG [3], and RAM [8, 9] in the present study.

\section{Clinical Characteristics of Final Analyzed Group of 99 Patients}

Significant differences were observed in etiology of basal liver diseases, serum levels of total bilirubin, prothrombin time, AFP at introducing LEN, maximum size of intrahepatic tumor, TNM-LCSGJ at introducing LEN, ECOG PS at time of PD, AFP at time of PD (before or after 1-month PD), and BCLC stage at time of PD between the $\mathrm{A}$ and $\mathrm{B}$ groups (Table 1). Postprogression treatment in the A group was best supportive care in 17, sorafenib in 4 , regorafenib in 3, ramucirumab in 1 , and hepatic arterial infusion chemotherapy in 1 (online suppl. Table 1). Of the 9 patients, who received postprogression therapies, in the A group, 8 showed PD and 1 had no evaluation at the time of analysis. On the other hand, 6 were treated with dose-up, 59 with the same dose, and 8 with more reduced dose after PD in the B group. Except for patients received combination with other therapeutic modalities
(RFA 1 , TACE 3 , or HAIC 2$)(n=6)$ and 1 patient without following imaging data $(n=1)$, only 2 showed no progression after PD by the same dose, while 64 showed continuous progression.

\section{Adverse Events}

AEs during LEN treatment are shown in Table 2. Although only HFSR was more frequent in the B group than the other (grade 0:1:2:3 = 44:13:16:0 vs. 22:2:1:1. $p=$ $0.019)$, there were no significant differences in other AEs (Table 2).

\section{Administration Period of LEN, Relative Changes in ALBI Score, and Therapeutic Responses}

There was no significant difference for TTP between the $\mathrm{A}$ and $\mathrm{B}$ groups (median 4.0 vs. 6.0 months, $p=0.514$ ). The LEN administration period in the A group was shorter (median 4.5 vs. 12.6 months, $p<0.001$ ) (Fig. 2a, b). Best therapeutic response by RECIST ( $p=0.862$ ), ALBI score at the time of $\mathrm{PD}(p=0.467)$, frequency of Child-Pugh class A at time of PD ( $p=0.199)$, decline of ECOG at time of PD from baseline $(p=0.141)$, and relative change in ALBI score from the baseline at the time of PD (delta ALBI score) $(p=0.691)$ did not show significant differences between the groups. In addition, PD patterns also did not show a significant difference between both groups $(p=0.939)($ Table 1$)$. 


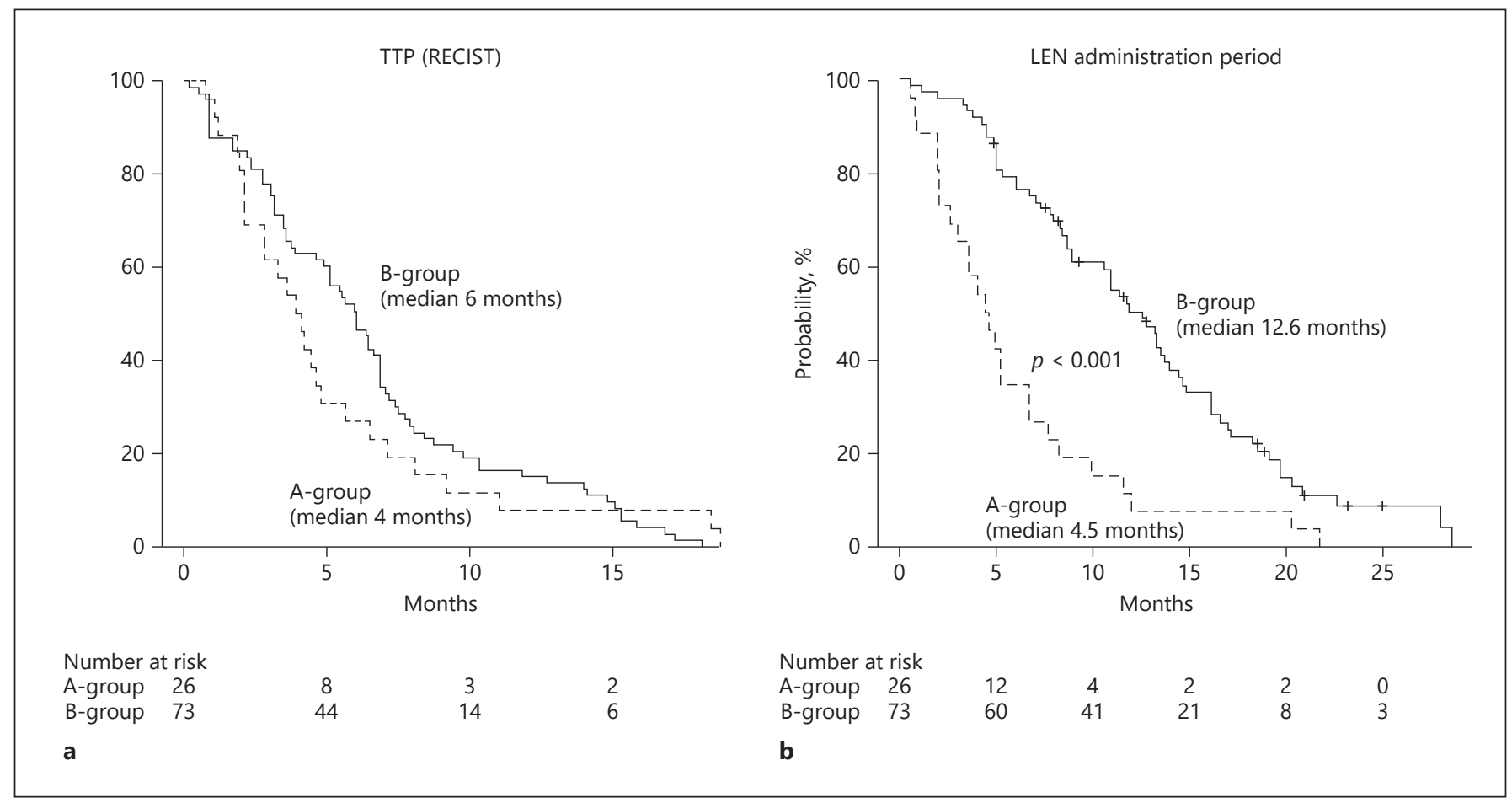

Fig. 2. a TTP shown by RECIST 1.1. b Period of lenvatinib administration. TTP, time to progression; RECIST, Response Evaluation Criteria In Solid Tumors version 1.1.

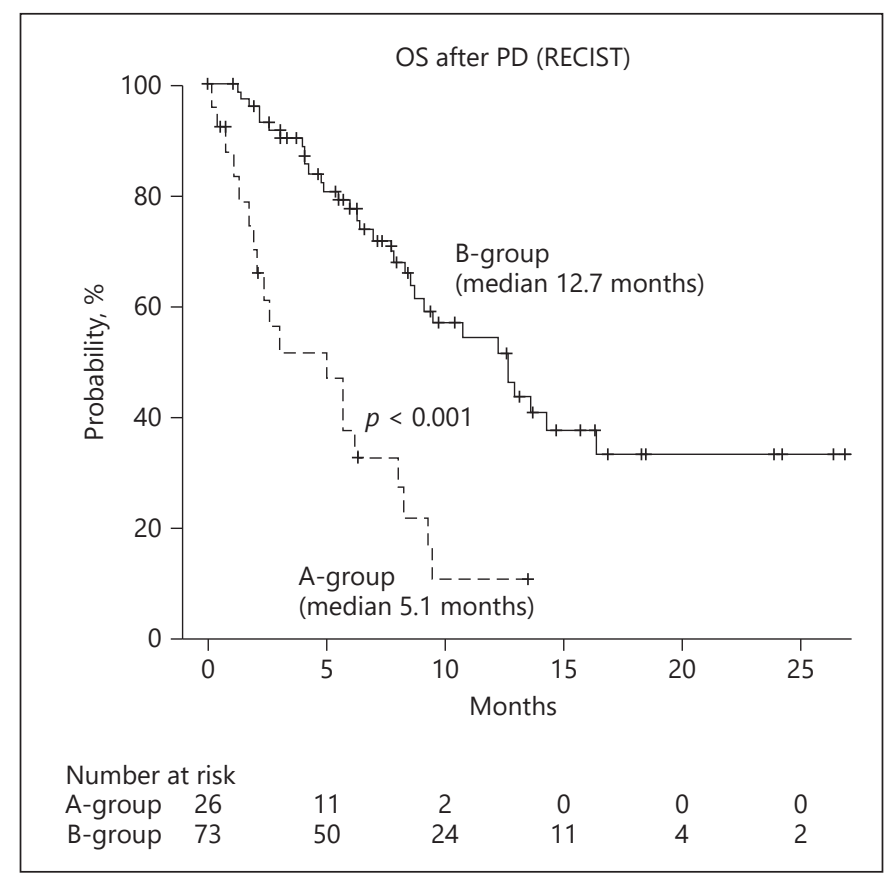

Fig. 3. OS after first PD, shown by RECIST 1.1. (A group: dotted line, B group: solid line). OS, overall survival; PD, progression of disease; RECIST, Response Evaluation Criteria In Solid Tumors version 1.1.

\section{OS after $P D$}

Of the $99 \mathrm{u}-\mathrm{HCC}$ patients analyzed, 52 died during the observation period (A vs. B group $=73.1$ vs. $45.2 \%$, $p=0.021$ ) (Table 1). OS after PD was better in the B group as compared to the A group (12.7 vs. 5.1 months, $p<0.001$ ) (Fig. 3). In the A group, although when the same analysis was performed after adjusting with IPW, the $\mathrm{B}$ group showed better prognosis in regard to OS after PD (10.8 vs. 5.8 months, $p<0.001$ ) (Fig. 4a). As a result, OS after introducing LEN was also better in the B group than the A group (19.6 vs. 11.2 months, $p<$ 0.001) (Fig. 4b).

In Cox hazard univariate analysis after adjustments with IPW, prognostic factors for OS following PD were mALBI $2 b$ or 3 at the time of PD (HR 2.160, $p=0.008$ ), decline of ECOG PS from baseline at time of PD (HR $3.578, p<0.001)$, elevated AFP $(\geq 100 \mathrm{ng} / \mathrm{mL})$ at time of introducing LEN (HR 1.804, $p=0.047$ ), PD pattern (appearance of new EHM) (HR 2.105, $p=0.013)$, positive for HFSR before PD (any grade) (HR 0.370, $p=0.002$ ), and continuing LEN beyond PD (HR 0.444, $p=0.014$ ), while mALBI $2 \mathrm{~b}$ or 3 at the time of PD (HR 1.983, $p=$ 0.021), decline of ECOG PS from baseline at time of PD 


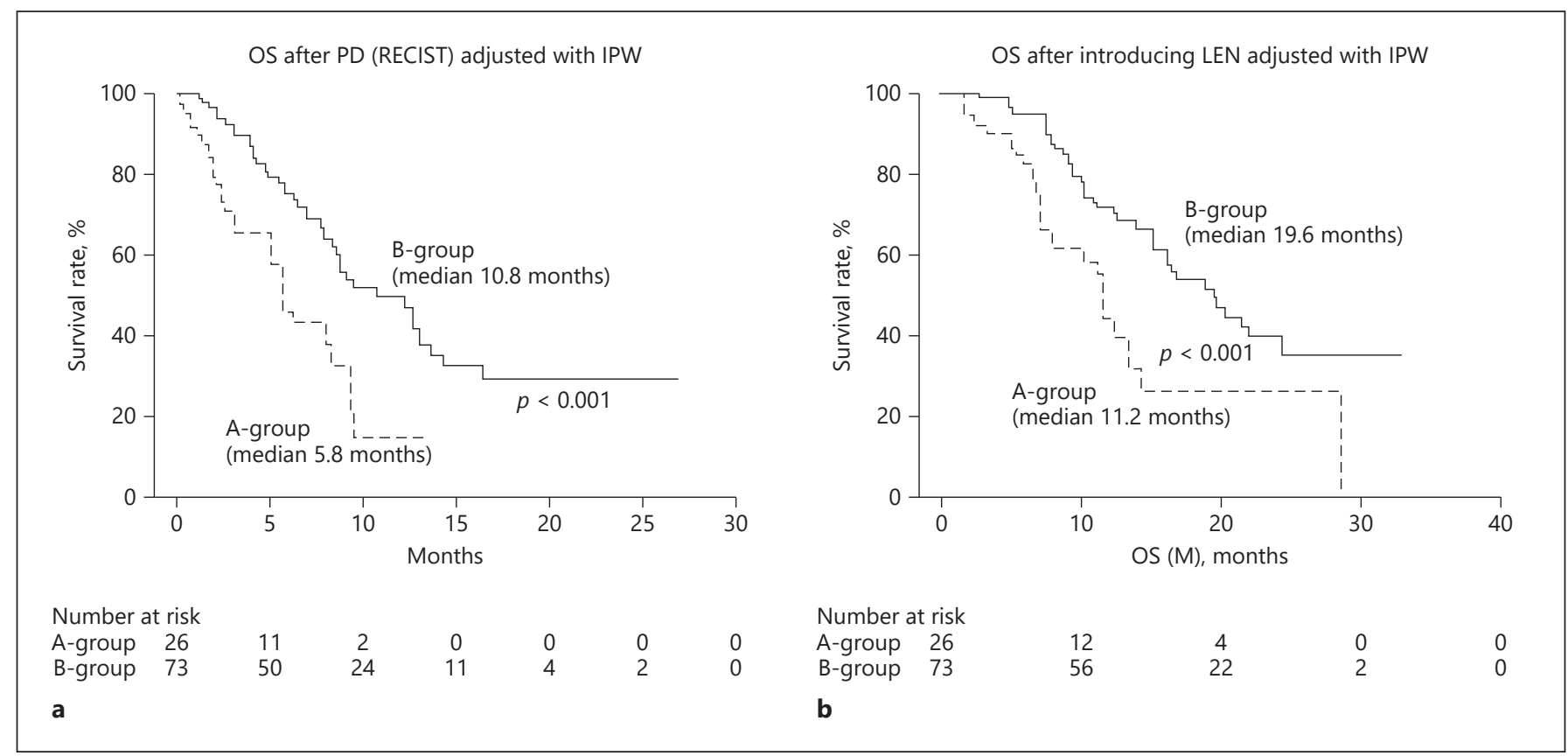

Fig. 4. OS after first PD by RECIST 1.1 following adjustment with IPW (a) and OS after introducing lenvatinib, adjusted with IPW (b) (A group: dotted line, B group: solid line). OS, overall survival; PD, progression of disease; IPW, inverse probability weighting; RECIST, Response Evaluation Criteria In Solid Tumors version 1.1.

(HR 3.180, $p<0.001)$, elevated AFP $(\geq 100 \mathrm{ng} / \mathrm{mL})$ at time of introducing LEN (HR 2.511, $p=0.004$ ), PD pattern (appearance of new extrahepatic metastasis) (HR 2.396, $p=0.006$ ), positive for HFSR before PD (any grade) (HR $0.292, p<0.001$ ), and continuing LEN beyond PD (HR $0.297, p<0.001$ ) were significant factors in multivariate analysis (Table 3). When OS after PD was evaluated in patients with Child-Pugh class A and ECOG PS 0/1 at time of $\mathrm{PD}$, the $\mathrm{B}$ group showed better OS after PD than the other (MST 13.0 vs. 8.1 months, $p=0.027$ ) (online suppl. Fig. 2). In the patients with Child-Pugh $\mathrm{A}$ and ECOG PS $0 / 1$ at time of PD of the A group, although 8 patients, who recieved post progression treatments $(n=8)$, showed longer median OS than those with BSC ( $n=7$ ) ( 8.8 vs. 5.1 months, respectively), there was no significant difference statistically $(p=0.324)$. In the patients with Child-Pugh A and ECOG PS 0/1 at time of PD of the A group, although 8 patients, who recieved post progression treatments $(n=8)$, showed longer median OS than those with BSC $(n=7)$ ( 8.8 vs. 5.1 months, respectively), there was no significant difference statistically $(p=0.324)$.

\section{Discussion}

In the present study, clinical factors and prognosis at the time of PD in u-HCC patients treated with LEN were assessed. Interestingly, the prognosis of $\mathrm{u}-\mathrm{HCC}$ patients for whom LEN treatment was discontinued after PD (A group) was significantly worse as compared to those who continued LEN beyond PD (B group), with the same result observed following adjustment with IPW. Our results showed that mALBI ( $2 \mathrm{~b}$ or 3 ) at time of PD, decline of ECOG PS from baseline at time of PD, elevated AFP $(\geq 100 \mathrm{ng} / \mathrm{mL})$ at time of introducing LEN, PD pattern (appearance of new EHM), positive for HFSR (any grade), and continuing LEN beyond PD were significant prognostic factors for OS following PD. Although Fu et al. [30] reported that BCLC-B $(p=0.002)$ and intrahepatic $\mathrm{PD}(p=0.024)$ were significantly correlated with post-disease progression OS in SOR treatment, BCLC stage and intrahepatic PD patterns were not significant prognostic factors in the present study. Of course, it is reasonable that malignant potential of tumors (elevated tumor marker), worse hepatic function, and decline of ECOG PS were negative prognostic factors for survival. In addition, the present results showed that AE of HFSR 
Table 3. Cox hazard analysis of characteristics and prognostic factors for overall survival after PD by RECIST 1.1 adjusted with IPW in u-HCC patients $(N=99)$

\begin{tabular}{|c|c|c|c|c|c|c|}
\hline & HR & $\begin{array}{l}\text { Univariate } \\
\text { analysis } \\
95 \% \mathrm{CI}\end{array}$ & $p$ value & HR & $\begin{array}{l}\text { Multivariate } \\
\text { analysis } \\
95 \% \text { CI }\end{array}$ & $p$ value \\
\hline Elderly (age $\geq 75$ years) & 1.363 & $0.789-2.355$ & 0.266 & & & \\
\hline Female gender & 1.576 & $0.878-2.829$ & 0.128 & & & \\
\hline Negative for hepatitis virus & 1.508 & $0.791-2.878$ & 0.213 & & & \\
\hline Started with reduced dose of LEN & 1.262 & $0.610-2.610$ & 0.531 & & & \\
\hline mALBI grade $2 \mathrm{~b} / 3$ (ALBI score $>-2.27$ ) at introduction of LEN & 1.519 & $0.902-2.557$ & 0.116 & & & \\
\hline mALBI grade $2 b / 3$ (ALBI score $>-2.27$ ) at time of PD & 2.160 & $1.218-3.829$ & 0.008 & 1.983 & $1.109-3.545$ & 0.021 \\
\hline ECOG PS 1 at introduction of LEN & 1.181 & $0.419-3.333$ & 0.753 & & & \\
\hline Decline of ECOG PS from baseline at time of PD & 3.578 & $2.010-6.365$ & $<0.001$ & 3.180 & $1.722-5.872$ & $<0.001$ \\
\hline BCLC-C at time of LEN introduction & 1.323 & $0.754-2.323$ & 0.329 & & & \\
\hline BCLC-C/D at time of PD & 1.296 & $0.722-2.328$ & 0.386 & & & \\
\hline $\mathrm{AFP} \geq 100 \mathrm{ng} / \mathrm{mL}$ at time of LEN introduction & 1.804 & $1.008-3.229$ & 0.047 & 2.511 & $1.347-4.679$ & 0.004 \\
\hline $\mathrm{AFP} \geq 100 \mathrm{ng} / \mathrm{mL}$ at time of $\mathrm{PD}$ & 1.608 & $0.884-2.924$ & 0.120 & & & \\
\hline Positive for history of MTA treatment & 1.275 & $0.726-2.237$ & 0.398 & & & \\
\hline No response to LEN & 1.444 & $0.853-2.444$ & 0.172 & & & \\
\hline PD pattern, appearance of new EHM & 2.105 & $1.173-3.777$ & 0.013 & 2.396 & $1.291-4.449$ & 0.006 \\
\hline PD pattern, appearance of MVI & 0.273 & $0.042-1.787$ & 0.176 & & & \\
\hline PD pattern, appearance of intrahepatic new lesion & 1.047 & $0.610-1.797$ & 0.868 & & & \\
\hline PD pattern, increasing size without new lesion & 0.859 & $0.486-1.517$ & 0.600 & & & \\
\hline HFSR (any grade) before PD & 0.370 & $0.200-0.685$ & 0.002 & 0.292 & $0.151-0.566$ & $<0.001$ \\
\hline LEN continued beyond PD & 0.444 & $0.232-0.848$ & 0.014 & 0.297 & $0.163-0.541$ & $<0.001$ \\
\hline
\end{tabular}

u-HCC, unresectable hepatocellular carcinoma; IPW, inverse probability weighting; $\mathrm{HR}$, hazard ratio; CI, confidential interval; $\mathrm{HCV}$, hepatitis C virus; LEN, lenvatinib; mALBI, modified albumin-bilirubin grade; BCLC-C, Barcelona Clinic Liver Cancer stage C; AFP, alpha-fetoprotein; MTA, molecular targeting agent; EHM, extrahepatic metastasis; MVI, major portal vein tumor thrombosis (Vp3 and Vp4); HFSR, hand-foot skin reaction; PD, progression of disease.

(any grade) and continuing LEN beyond PD were positive prognostic factors for survival after first PD. Regarding HFSR, it has been reported as a positive prognostic factor for better prognosis in SOR treatment [31] as well as LEN treatment [6]. Moreover, in previous studies, $\mathrm{Mi}$ yahara et al. [32] and Wada et al. [33] analyzed continuation of SOR treatment beyond first PD confirmation before development of second-line chemotherapy, respectively. With no present establishment of effective postprogression therapy after LEN failure and when no rapid progression is noted during LEN treatment and control of $\mathrm{AE}$ is obtained in u-HCC patients whose ECOG PS and hepatic function permit, our data suggest that continuation of that beyond PD might be a reasonable therapeutic option. In SOR treatment, Miyahara et al. [32] reported that the patients, who stopped SOR at $\mathrm{PD}$, showed increasing tumor growth, while those who continued SOR beyond first PD did not $(p=0.002)$. Although the present database has few data concerning with growth rates of tumors, especially of the A group, and there was a continuous tendency of PD after first PD was observed in many patients of the B group, continuing LEN beyond $\mathrm{PD}$ also might play a role in curbing rapid progression. Nevertheless, based on the present results, establishment of effective postprogression modalities following LEN failure is urgently needed.

There is increasing recognition regarding the clinical importance of MTA sequential treatment for improving the prognosis of u-HCC patients. Since introduction of REG as second-line treatment for SOR, it has been reported that MTA sequential treatment has a greater contribution to improving prognosis as compared to single MTA line treatment [34]. LEN was recently developed as a first-line MTA drug [5], though it also serves as a second- and even third-line treatment option in Japan [7, 35-39]. We previously reported that the total MTA administration period including LEN treatment had a good correlation with OS after introduction of the initial MTA drug (SOR in $95.2 \%$ of those patients) ( $r=0.946,95 \%$ CI: $0.918-0.965, p<0.001)$, with a median OS after that in- 
troduction of 46.2 months [40]. Those results suggest that SOR has effective postprogression treatment options. In contrast, no effective postprogression treatment has been established after LEN treatment failure, which is an important unmet clinical need. In the REFLECT trial, patients treated with LEN showed significantly better progression-free survival (PFS) as compared to those treated with SOR (7.4 vs. 3.7 months; HR 0.66, 95\% CI: 0.57$0.77)$ [5]. However, even though SOR was available as a postprogression option in patients treated with LEN in that trial, LEN did not show superiority to SOR in regard to OS (median 13.6 vs. 12.3 months; HR $0.92,95 \% \mathrm{CI}$ : 0.79-1.06) [5]. In another study of 25 patients treated with SOR after LEN failure, a poor disease control rate shown by mRECIST (16.0\%) and PFS (1.8 months) was reported [41]. A future analysis is needed to elucidate which order of sequential treatment is better, SOR followed by LEN or LEN by SOR.

A previous study found that the percentage of patients given LEN as first-line treatment and who maintained Child-Pugh A, in whom RAM was indicated, was low (approximately $20 \%$ ) at the time of LEN failure [42]; thus, no conclusions could be reached regarding whether ramucirumab is an effective next-line treatment following LEN. Kuzuya reported good therapeutic usefulness following LEN failure in $12 \mathrm{u}$-HCC patients (disease control rate [DCR]: 80\%) [43], whereas the results presented by our group were not similar in $28 \mathrm{u}$-HCC patients under the same situation (DCR: 42.3\%) [44]. Future analysis for therapeutic effect of RAM after LEN failure will be needed. In any case, based on results of animal experimentation, Yang et al. [45] suggested that host hepatocyte but not tumor cellderived VEGF is responsible for facilitating cancer metastasis mechanistically and proposed that nonstop persistent anti-VEGF therapy be given as treatment for humans with cancer. To improve prognosis more in u-HCC patients treated with LEN, urgent development of effective MTA drugs following LEN failure is a crucial need.

In cases with transarterial catheter chemoembolization (TACE) refractoriness [46] or those unsuitable for TACE [47], clinical consensus indicates that switching to MTA treatment should be considered. In addition to a recent clinical trend of HCC treatment based on development of MTA drugs, combination treatment with atezolizumab and bevacizumab has been approved in September 2020 as a new first-line option, as that combination resulted in better OS and PFS as compared to sorafenib (median PFS: 6.8 vs. 4.3 months, HR 0.59, 95\% CI: $0.47-$ 0.76 [ $p<0.001$ ]; OS at 12 months: 67.2 vs. $54.6 \%$, HR 0.58 , 95\% CI: $0.42-0.79$ [ $p<0.001]$ ] [48]. Determination of a

An Unmet Need in Lenvatinib Treatment better order of systemic chemotherapy agents to contribute to improved prognosis will become important for $\mathrm{u}$ HCC treatment in clinical practice.

The present study has some limitations. First, even though this was a multicenter analysis, it was performed in a retrospective manner. Also, the number of patients analyzed was small. Finally, there were no defined criteria used for continuing LEN treatment or determining cessation of beyond PD treatment with LEN. A future study focusing on establishment of postprogression treatment after LEN failure, especially with a larger number of patients given LEN, is needed. Since it will be difficult to perform a randomized control study, a prospective observational study at minimum should be planned in the near future.

Although LEN is a powerful drug that serves not only as a first- but also as a second- and third-line option for treatment of $\mathrm{u}$-HCC in the real-world clinical practice, the present results indicate that it might be better positioned as a late-line drug in selected patients with clinical reserves, such as ALBI grade 1 liver function or a nonaggressive tumor. Furthermore, when there is no decline of ECOG PS from baseline during LEN treatment and hepatic reserve function permits, it may be better to continue LEN treatment beyond PD, especially in u-HCC patients showed HFSR during LEN treatment, at least until a more effective drug as a postprogression treatment after LEN failure is developed. In any case, urgent development of effective drugs following LEN failure is an urgent unmet clinical need.

\section{Statement of Ethics}

The protocol of the present study was approved by the Institutional Ethics Committee of Ehime Prefectural Central Hospital (IRB No. 30-66), and the research was conducted ethically in accordance with the World Medical Association Declaration of Helsinki. Written patient consent was obtained from all patients.

\section{Conflict of Interest Statement}

Atsushi Hiraoka, MD, PhD: lecture fees from Bayer, Eisai, and Otsuka. Takashi Kumada, MD, PhD: lecture fees from Eisai. Masatoshi Kudo, MD, PhD: advisory role with Eisai, Ono, MSD, Bristol Myers Squibb, and Roche; lecture fees from Eisai, Bayer, MSD, Bristol Myers Squibb, Eli Lilly, and EA Pharma; and research funding from Gilead Sciences, Taiho, Sumitomo Dainippon Pharma, Takeda, Otsuka, EA Pharma, AbbVie, and Eisai. None of the other authors have potential conflicts of interest to declare.

\section{Funding Sources}

The authors did not receive any funding. 


\section{Author Contributions}

A.H., T.K., M.H., K.Ts., E.I., N.S., H.S., S.Y., H.T., Y.K., Y.H., and M.Ku. conceived the study and participated in its design and coordination. A.H., T.K., T.T., K.ka., J.T., S.F., M.A., T.I., K.Tak.,
K.Taj., H.Oc., K.K., H.Oh., K.N., A.T., T.N., N.I., K.H., T.A., M.I., S.N., K.M., K.J., and M.K. performed data curation. A.H. performed statistical analyses and interpretation. A.H. and T.K. drafted the text. All authors have read and approved the final version of the manuscript.

\section{References}

1 Parkin DM, Bray F, Ferlay J, Pisani P. Estimating the world cancer burden: Globocan 2000 Int J Cancer. 2001 Oct 15;94(2):153-6.

2 Llovet JM, Ricci S, Mazzaferro V, Hilgard P, Gane E, Blanc JF, et al. Sorafenib in advanced hepatocellular carcinoma. N Engl J Med. 2008 Jul 24;359(4):378-90.

3 Bruix J, Qin S, Merle P, Granito A, Huang YH, Bodoky G, et al. Regorafenib for patients with hepatocellular carcinoma who progressed on sorafenib treatment (RESORCE): a randomised, double-blind, placebo-controlled, phase 3 trial. Lancet. 2017 Jan 7;389(10064): $56-66$.

4 Terashima T, Yamashita T, Takata N, Nakagawa $\mathrm{H}$, Toyama $\mathrm{T}$, Arai $\mathrm{K}$, et al. Post-progression survival and progression-free survival in patients with advanced hepatocellular carcinoma treated by sorafenib. Hepatol Res. 2016 Jun;46(7):650-6.

5 Kudo M, Finn RS, Qin S, Han K-H, Ikeda $\mathrm{K}$, Piscaglia $\mathrm{F}$, et al. Lenvatinib versus sorafenib in first-line treatment of patients with unresectable hepatocellular carcinoma: a randomised phase 3 non-inferiority trial. Lancet. 2018 Feb 9;391(10126):116373.

6 Hiraoka A, Kumada T, Atsukawa M, Hirooka M, Tsuji K, Ishikawa T, et al. Prognostic factor of lenvatinib for unresectable hepatocellular carcinoma in real-world conditions: multicenter analysis. Cancer Med. 2019 Jul;8(8): 3719-28.

7 Hiraoka A, Kumada T, Kariyama K, Takaguchi K, Itobayashi E, Shimada N, et al. Therapeutic potential of lenvatinib for unresectable hepatocellular carcinoma in clinical practice: multicenter analysis. Hepatol Res. 2019 Jan; 49(1):111-7.

8 Zhu AX, Finn RS, Galle PR, Llovet JM, Kudo M. Ramucirumab in advanced hepatocellular carcinoma in REACH-2: the true value of alpha-fetoprotein. Lancet Oncol. 2019 Apr; 20(4):e191.

9 Kudo M, Okusaka T, Motomura K, Ohno I, Morimoto M, Seo S, et al. Ramucirumab after prior sorafenib in patients with advanced hepatocellular carcinoma and elevated alphafetoprotein: Japanese subgroup analysis of the REACH-2 trial. J Gastroenterol. 2020 Jun; 55(6):627-39.

10 Eisenhauer EA, Therasse P, Bogaerts J, Schwartz LH, Sargent D, Ford R, et al. New response evaluation criteria in solid tumours: revised RECIST guideline (version 1.1). Eur J Cancer. 2009 Jan;45(2):228-47.
11 Lencioni R, Llovet JM. Modified RECIST (mRECIST) assessment for hepatocellular carcinoma. Semin Liver Dis. 2010 Feb;30(1):52-60.

12 Pugh RN, Murray-Lyon IM, Dawson JL, Pietroni MC, Williams R. Transection of the oesophagus for bleeding oesophageal varices. $\mathrm{Br}$ J Surg. 1973 Aug;60(8):646-9.

13 Johnson PJ, Berhane S, Kagebayashi C, Satomura S, Teng M, Reeves HL, et al. Assessment of liver function in patients with hepatocellular carcinoma: a new evidence-based approach-the ALBI grade. J Clin Oncol. 2015 Feb 20;33(6):550-8.

14 Hiraoka A, Kumada T, Michitaka K, Toyoda $\mathrm{H}$, Tada T, Ueki H, et al. Usefulness of albumin-bilirubin grade for evaluation of prognosis of 2584 Japanese patients with hepatocellular carcinoma. J Gastroenterol Hepatol. 2016 May;31(5):1031-6.

15 Hiraoka A, Kumada T, Kudo M, Hirooka M, Tsuji K, Itobayashi E, et al. Albumin-bilirubin (ALBI) grade as part of the evidence-based clinical practice guideline for HCC of the Japan Society of Hepatology: a comparison with the liver damage and Child-Pugh classifications. Liver Cancer. 2017 Jun;6(3):204-15.

16 Hiraoka A, Michitaka K, Kumada T, Izumi N, Kadoya M, Kokudo N, et al. Validation and potential of albumin-bilirubin grade and prognostication in a nationwide survey of 46,681 hepatocellular carcinoma patients in Japan: the need for a more detailed evaluation of hepatic function. Liver Cancer. 2017 Nov;6(4):325-36.

17 Hiraoka A, Kumada T, Tsuji K, Takaguchi K, Itobayashi E, Kariyama K, et al. Validation of modified ALBI grade for more detailed assessment of hepatic function in hepatocellular carcinoma patients: a multicenter analysis. Liver Cancer. 2019 Mar;8(2):121-9.

18 Bruix J, Sherman M. Management of hepatocellular carcinoma. Hepatology. 2005 Nov; 42(5):1208-36.

19 Di Martino M, Marin D, Guerrisi A, Baski M, Galati F, Rossi M, et al. Intraindividual comparison of gadoxetate disodium-enhanced MR imaging and 64-section multidetector CT in the detection of hepatocellular carcinoma in patients with cirrhosis. Radiology. 2010 Sep;256(3):806-16.

20 Sano K, Ichikawa T, Motosugi U, Sou H, Muhi AM, Matsuda M, et al. Imaging study of early hepatocellular carcinoma: usefulness of gadoxetic acid-enhanced MR imaging. Radiology. 2011 Dec;261(3):834-44.

21 Hiraoka A, Ichiryu M, Tazuya N, Ochi H, Tanabe A, Nakahara $\mathrm{H}$, et al. Clinical translation in the treatment of hepatocellular carcinoma following the introduction of contrast-enhanced ultrasonography with Sonazoid. Oncol Lett. 2010 Jan;1(1):57-61.

22 Hiraoka A, Hiasa Y, Onji M, Michitaka K. New contrast enhanced ultrasonography agent: impact of Sonazoid on radiofrequency ablation. J Gastroenterol Hepatol. 2011 Apr; 26(4):616-8.

23 Forner A, Reig M, Bruix J. Hepatocellular carcinoma. Lancet. 2018 Mar 31;391(10127): 1301-14.

24 The Liver Cancer Study Group of Japan. The general rules for the clinical and pathological study of primary liver cancer. 6th ed. Tokyo: Kanehara; 2015. p. 26.

25 National, Cancer, Institutie. Available from: https://ctepcancergov/protocolDevelopment/adverse_effectshtmA ccessed 2020 Jun 30.

26 Xie J, Liu C. Adjusted Kaplan-Meier estimator and log-rank test with inverse probability of treatment weighting for survival data. Stat Med. 2005 Oct 30;24(20):3089-110.

27 Kim HT. Cumulative incidence in competing risks data and competing risks regression analysis. Clin Cancer Res. 2007 Jan 15;13(2 Pt 1):559-65.

28 Kanda Y. Investigation of the freely available easy-to-use software 'EZR' for medical statistics. Bone Marrow Transplant. 2013 Mar; 48(3):452-8.

29 Kaneko S, Tsuchiya K, Kurosaki M, Kirino S, Inada K, Yamashita K, et al. Three criteria for radiological response on survival in patients with hepatocellular carcinoma treated with lenvatinib. Hepatol Res. 2020 Jan;50(1):137-43.

$30 \mathrm{Fu}$ SR, Zhang YQ, Li Y, Hu BS, He X, Huang JW, et al. Sorafenib continuation after first disease progression could reduce disease flares and provide survival benefits in patients with hepatocellular carcinoma: a pilot retrospective study. Asian Pac J Cancer Prev. 2014; 15(7):3151-6.

31 Ogawa C, Morita M, Omura A, Noda T, Kubo A, Matsunaka T, et al. Hand-foot syndrome and post-progression treatment are the good predictors of better survival in advanced hepatocellular carcinoma treated with sorafenib: a multicenter study. Oncology. 2017;93(Suppl 1):113-9.

32 Miyahara K, Nouso K, Morimoto Y, Takeuchi Y, Hagihara H, Kuwaki K, et al. Efficacy of sorafenib beyond first progression in patients with metastatic hepatocellular carcinoma. Hepatol Res. 2014 Mar;44(3):296-301. 
33 Wada Y, Takami Y, Tateishi M, Ryu T, Mikagi K, Saitsu H. The efficacy of continued sorafenib treatment after radiologic confirmation of progressive disease in patients with advanced hepatocellular carcinoma. PLoS One. 2016;11(1):e0146456.

34 Finn RS, Merle P, Granito A, Huang YH, Bodoky G, Pracht M, et al. Outcomes of sequential treatment with sorafenib followed by regorafenib for HCC: additional analyses from the phase III RESORCE trial. J Hepatol. 2018 Aug;69(2):353-8.

35 Hiraoka A, Kumada T, Kariyama K, Takaguchi K, Atsukawa M, Itobayashi E, et al. Clinical features of lenvatinib for unresectable hepatocellular carcinoma in real-world conditions: multicenter analysis. Cancer Med. 2019 Jan;8(1):137-46.

36 Fuchigami A, Imai $Y$, Uchida $Y$, Uchiya $H$, Fujii Y, Nakazawa M, et al. Therapeutic efficacy of lenvatinib for patients with unresectable hepatocellular carcinoma based on the middle-term outcome. PLoS One. 2020;15(4): e0231427.

37 Ogasawara S, Ooka Y, Itokawa N, Inoue M, Okabe S, Seki A, et al. Sequential therapy with sorafenib and regorafenib for advanced hepatocellular carcinoma: a multicenter retrospective study in Japan. Invest New Drugs. 2020 Feb;38(1):172-80.
38 Sho T, Suda G, Ogawa K, Kimura M, Shimazaki T, Maehara O, et al. Early response and safety of lenvatinib for patients with advanced hepatocellular carcinoma in a real-world setting. JGH Open. 2020 Feb;4(1):54-60.

39 Takahashi A, Moriguchi M, Seko Y, Shima T, Mitsumoto Y, Takashima H, et al. Early tumor shrinkage as a predictive factor for outcomes in hepatocellular carcinoma patients treated with lenvatinib: a multicenter analysis. Cancers. 2020 Mar 23;12(3):754.

40 Hiraoka A, Kumada T, Atsukawa M, Hirooka M, Tsuji K, Ishikawa T, et al. Important clinical factors in sequential therapy including lenvatinib against unresectable hepatocellular carcinoma. Oncology. 2019;97(5):277-85.

41 Maruta S. Post-treatments of lenvatinib in patients with advanced hepatocellular carcinoma. Hepatol Int. 2020;14(Suppl 1):S234-35.

42 Hiraoka A, Kumada T, Fukunishi S, Atsukawa M, Hirooka M, Tsuji K, et al. Post-progression treatment eligibility of unresectable hepatocellular carcinoma patients treated with lenvatinib. Liver Cancer. 2020 Jan;9(1):7383.

43 Kuzuya T, Ishigami M, Ito T, Ishizu Y, Honda $\mathrm{T}$, Ishikawa $\mathrm{T}$, et al. Initial experience of ramucirumab treatment after lenvatinib failure for patients with advanced hepatocellular carcinoma. Anticancer Res. 2020 Apr;40(4): 2089-93.
44 Hiraoka A, Kumada T, Tada T, Ogawa C, Tani J, Fukunishi S, et al. Therapeutic efficacy of ramucirumab after lenvatinib for post-progression treatment of unresectable hepatocellular carcinoma. Gastroenterol Rep. 2020.

45 Yang Y, Zhang Y, Iwamoto H, Hosaka K, Seki $\mathrm{T}$, Andersson P, et al. Discontinuation of antiVEGF cancer therapy promotes metastasis through a liver revascularization mechanism. Nat Commun. 2016 Sep 1;7:12680.

46 Kudo M, Matsui O, Izumi N, Kadoya M, Okusaka T, Miyayama S, et al. Transarterial chemoembolization failure/refractoriness: $\mathrm{JSH}$ LCSGJ criteria 2014 update. Oncology. 2014; 87(Suppl 1):22-31.

47 Kudo M, Han KH, Ye SL, Zhou J, Huang YH, Lin SM, et al. A changing paradigm for the treatment of intermediate-stage hepatocellular carcinoma: Asia-Pacific primary liver cancer expert consensus statements. Liver Cancer. 2020 Jun;9(3):245-60.

48 Finn RS, Qin S, Ikeda M, Galle PR, Ducreux M, Kim T-Y, et al. Atezolizumab plus bevacizumab in unresectable hepatocellular carcinoma. N Engl J Med. 2020 May 14;382(20): 1894-905. 